\title{
Navegação autônoma de múltiplos robôs baseada em realidade aumentada ${ }^{\star}$
}

\author{
João Paulo Lima Silva de Almeida ${ }^{*, * *}$ \\ Renan Taizo Nakashima ${ }^{* *}$ Flávio Neves-Jr ${ }^{* *}$ \\ Lúcia Valéria Ramos de Arruda** \\ * Instituto Federal do Paraná (IFPR), Jacarezinho, Paraná, Brasil \\ (e-mail: joao.almeida@ifpr.edu.br). \\ ** Universidade Tecnológica Federal do Paraná (UTFPR), Curitiba, \\ Paraná, Brasil (e-mail: nakashima@alunos.utfpr.edu.br e \\ $\{$ neves,lvrarruda\}@utfpr.edu.br)
}

\begin{abstract}
This work presents a platform based on augmented reality for simulation, analysis and test of navigation systems to multiple robots in a real lab-scale environment. The main objective of this platform is to provide conditions for the use of simple and low-cost robots, with hardware constraints, in autonomous navigation applications. The framework Robot Operating System (ROS) is used to integrate all platform's components. An application example is presented to demonstrate the available resources during experiments, where multiple real Sphero robots receive their navigation skills in a virtual and independent way, ensuring a decentralize navigation behavior. The achieved results confirm the feasibility of this experimental tool.

Resumo: Este trabalho apresenta uma plataforma baseada em realidade aumentada para simulação, análise e teste de sistemas de navegação para múltiplos robôs em um ambiente real, em escala de laboratório. O principal objetivo desta plataforma é fornecer condições para que robôs simples e de baixo custo, com limitações em seu hardware, possam ser utilizados em aplicações de navegação autônoma. O framework Robot Operating System (ROS) é usado para integrar todos componentes da plataforma. Um exemplo de aplicação é implementado para demonstrar os recursos disponíveis nos experimentos, em que múltiplos robôs reais do tipo Sphero recebem suas habilidades de navegação de forma virtual e independente, garantindo um comportamento descentralizado de navegação. Os resultados alcançados confirmam a factibilidade desta ferramenta experimental.
\end{abstract}

Keywords: Robotic; Multi-robot systems; Augmented Reality.

Palavras-chaves: Robótica; Sistemas multirrobô; Realidade aumentada.

\section{INTRODUÇÃO}

As pesquisas que envolvem os sistemas multirrobô (SMR) vem demonstrando avanços significativos no diversos segmentos da robótica móvel que suportam tarefas cooperativas, tais como exploração de ambientes desconhecidos em busca de pontos de interesse (Almeida et al., 2019), operações de busca e resgate (Yanguas-Rojas and MojicaNava, 2017), alocação de pontos de trabalho para múltiplos Automated Guided Vehicles (AGVs) em ambientes industriais (Han et al., 2017), entre outros.

O desenvolvimento de estratégias de coordenação de SMRs que levam à realização bem sucedida das tarefas acima mencionadas, desde o controle de movimento dos múltiplos robôs atingindo as camadas de planejamento de trajetória e distribuição de tarefas, requer etapas de testes e validação, muitas vezes realizadas por meio de simulações e / ou experimentos reais. Geralmente, os cenários simulados dispõem de ferramentas computacionais que flexibilizam

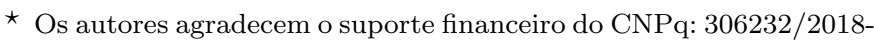
9 e 306599/2018-0.
}

a implementação de múltiplos robôs em diversas tarefas complexas e em situações controladas, facilitando o teste e validação dos SMRs em desenvolvimento. Por outro lado, os experimentos com robôs e outros dispositivos reais, inseridos em um ambiente real, mesmo em escala de laboratório, permitem a análise e avaliação do SMR em situações mais realistas, que incluem a presença de ruídos, falhas de comunicação, limitações físicas e de hardware dos robôs, entre outras (Reina et al., 2015).

Além desses problemas próprios aos experimentos reais, existem outros fatores que dificultam a validação de novas estratégias para SMR em situações reais, tais que o elevado custo e complexidade dos robôs e/ou seus dispositivos de hardware, especificados para a aplicação pretendida (Millard et al., 2018). Por este motivo, o desenvolvedor de (ou parte de) um SMR deve contar com um ambiente alternativo que possibilite a realização de experimentos combinando as caracteristicas de cenários simulados e reais. As abordagens experimentais baseadas em realidade mista para aplicações em robótica são o estado da arte para este propósito, onde elementos simulados são inseridos em ambientes com objetos reais (robôs reais, por exemplo) por 
meio de um sistema de realidade aumentada (Hönig et al., 2015).

Em (Millard et al., 2018), por exemplo, um ambiente em realidade aumentada é desenvolvido para facilitar a análise pelo usuário, de informações obtidas da navegação de múltiplos robôs reais. As arquiteturas baseadas em realidade aumentada também são utilizadas para implementar dispositivos virtuais de sensoriamento e comunicação em robôs reais (Kilobots e e-pucks) com capacidades limitadas (Reina et al., 2017, 2015). Por meio dos dispositivos virtuais, o usuário pode realizar testes e confirmar o funcionamento básico desses hardwares antes do processo de sua prototipagem ou aquisição.

Neste trabalho, é proposta uma plataforma baseada em realidade aumentada que suporta experimentos que envolvam múltiplos robôs reais com limitações nos sistemas de sensoriamento e comunicação, inseridos em um ambiente real em escala de laboratório. O principal objetivo da plataforma é possibilitar a implementação e testes de estratégias de coordenação de SMRs e também conferir virtualmente aos robôs as habilidades de sensoriamento, locomoção e comunicação requeridas para a realização das tarefas pretendidas, ou seja, os robôs utilizados nos experimentos conduzidos nesta plataforma não precisam ter os dispositivos reais para tais propósitos. Além disso, o sistema em realidade aumentada possibilita o usuário ter uma visão intuitiva, em tempo real, dos fatores que afetam o desempenho dos robôs.

A plataforma proposta neste artigo utiliza componentes comumente disponíveis para os projetistas e usuários de SMRs, tais como: um computador com o framework Robot Operating System ${ }^{1}$ (ROS), o qual é amplamente utilizado em aplicações da área de robótica devido ao seu funcionamento padronizado para a integração de diferentes dispositivos; uma câmera USB; marcadores visuais do tipo ARTag (Fiala, 2005); e robôs móveis com comunicação Bluetooth.

Para demonstrar o funcionamento desta plataforma, a estratégia de planejamento de trajetória apresentada em (Almeida et al., 2017) é implementada na plataforma desenvolvida. Nesta implementação, a camada de realidade aumentada é utilizada para guiar os robôs reais através de trajetórias calculadas, e para conferir, na ausência de sensores disponíveis, capacidade na detecção de obstáculos não previstos durante a navegação e assim realizar o ajuste de trajetória para evitar colisões e retormar a trajetória planejada após desvio.

Este trabalho é organizado da seguinte forma: uma descrição da plataforma baseada em realidade aumentada é apresentada na seção 2; o exemplo de aplicação é descrito na seção 3; os resultados são analisados na seção 4; e as principais conclusões são apresentadas na seção 5 .

\section{DESCRIÇÃO DA ARQUITETURA BASEADA EM REALIDADE AUMENTADA}

A plataforma baseada em realidade aumentada, proposta neste trabalho, possui os seguintes componentes: $(i)$ um ambiente real, retangular e plano; (ii) uma câmera USB,

\footnotetext{
1 http://wiki.ros.org
}

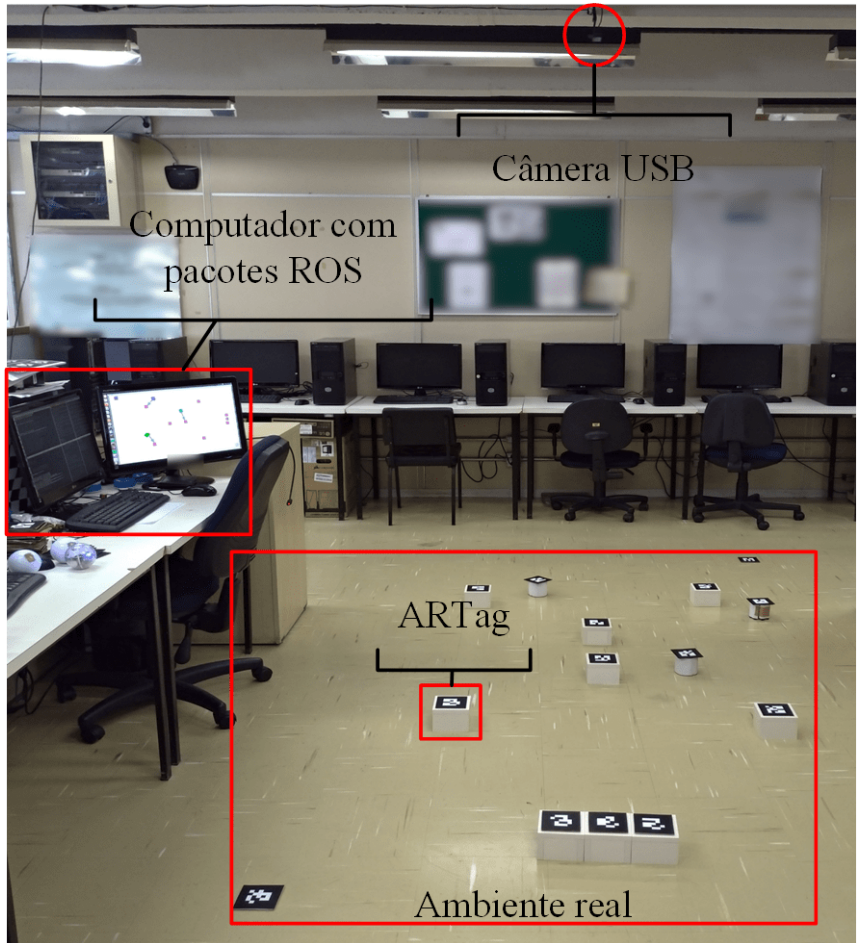

Figura 1. Ambiente em escala de laboratório usado nos experimentos.

posicionada acima deste ambiente; e (iii) um computador que suporta a execução do framework ROS. Tais componentes são apresentados na Figura 1.

De uma forma geral, os marcadores visuais ARTag (ambiente real da Figura 1) são utilizados para identificar todos os objetos inseridos no cenário (robôs e outros elementos). Esta identificação ocorre constantemente por meio de uma imagem digital capturada pela câmera e mostradas na camada virtual de realidade aumentada, implementada no computador e que será detalhada a seguir.

\subsection{Descrição dos robôs Sphero}

O SMR utilizado como exemplo nos experimentos desta arquitetura é composto por múltiplos robôs Sphero ${ }^{2}$ (Figura 2 (a)). Entretanto, qualquer tipo de robô móvel pode ser utilizado, desde que seu hardware permita a conectividade com o ROS, por meio de uma comunicação Bluetooth, e que sua estrutura tenha espaço para suportar um marcador visual do tipo ARTag e deixá-lo sempre visível à câmera, assim como no exemplo da Figura 1. No caso dos robôs Sphero, devido a seu formato esférico, um suporte é desenvolvido em uma impressora 3D, permitindo que as ARTags fiquem sempre visíveis à câmera, sem prejudicar os movimentos do robô. Este suporte é apresentado na Figura 2 (b).

Os robôs móveis Sphero possuem uma estrutura muito simples, revestida por um material plástico de formato esférico. Estes robôs são originalmente idealizados para serem comandados manualmente por um dispositivo móvel via Bluetooth, sendo capazes de se locomoverem no plano $(x, y)$, por meio da rotação de seu revestimento esférico.

\footnotetext{
2 http://www.sphero.com/sphero
} 


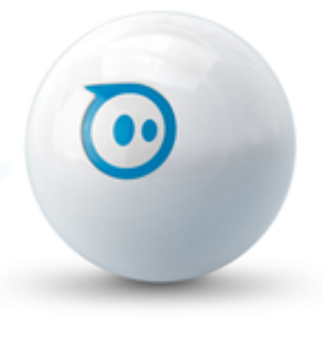

(a)

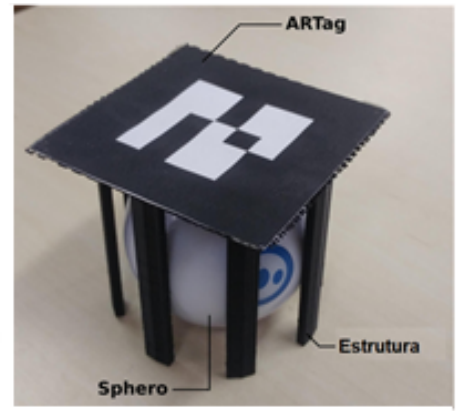

(b)
Figura 2. (a) Robô Sphero. (b) Robô com suporte da ARTag.

Além disso, estes robôs não possuem sensores para detecção de proximidade de objetos.

No entanto, um pacote ROS, denominado sphero_swarm ${ }^{3}$, é disponível para viabilizar a comunicação entre os robôs Sphero e o computador, com a possibilidade de enviar comandos de velocidade e orientação aos robôs.

Devido às suas poucas habilidades, estes robôs não são candidatos a formar um SMR em aplicações complexas, que requerem uma estratégia de controle avançada, combinadas a habilidades por exemplo, de detecção e identificação de obstáculos em região próxima.

Neste contexto, os robôs Sphero servirão para avaliar se a plataforma em realidade aumentada é capaz de fornecer virtualmente as habilidades necessárias para a execução do sistema de navegação autônomo descrito em (Almeida et al., 2019).

\subsection{Identificação dos objetos e camada virtual}

Todos os robôs e outros objetos considerados no ambiente são representados por meio de ARTags. Estes marcadores são detectados por uma imagem digital (obtida pela câmera USB) e identificados por meio do pacote ar_track_alvar ${ }^{4}$. Conforme isso ocorre, este pacote publica cada nome identificador e sua respectiva posição no ambiente em tópicos ROS específicos. Cada ARTag usada nesta plataforma possui uma dimensão de $94 \mathrm{~mm}$ x $94 \mathrm{~mm}$.

A partir dos dados de posição, cada robô é virtualmente dotado de um sistema autônomo de navegação, composto por um controle inteligente para sua locomoção e habilidades para detectar e identificar outros objetos (alvos e obstáculos). Este sistema de navegação corresponde a scripts individuais, desenvolvidos de acordo com o framework ActionLib ${ }^{5}$, que permite o processamento das tarefas de uma forma preemptiva. Portanto, cada robô executa seu próprio script para emular seus controladores de locomoção e suas habilidades de detecção, alcançando um comportamento descentralizado no SMR.

O controle de locomoção comanda o robô Sphero para que ele seja capaz de seguir uma trajetória até um ponto conhecido (alvo representado pela ARTag) e de realizar

\footnotetext{
3 https://github.com/darin-costello/sphero_swarm

4 http://wiki.ros.org/ar_track_alvar

5 http://wiki.ros.org/actionlib
}

manobras para o desvio de obstáculos, quando necessário. Para isto, os controladores fuzzy descritos em (Nakashima et al., 2018) são implementados para cara robô nesta plataforma. Basicamente, as ações destes controladores são combinadas por uma variável fuzzy de decisão $(\tau \in[0,1])$, que define se o robô deve seguir fielmente uma trajetória $(\tau \approx 1)$, desviar de um obstáculo com médio risco de colisão $(\tau \approx 0,5)$, ou desviar de um obstáculo com alto risco de colisão $(\tau \approx 0)$.

Em relação às habilidade de deteç̧ão, seu principal objetivo é conferir aos robôs a capacidade de detectar objetos ao seu redor e calcular o risco de colisão de cada elemento detectado desta forma, como ocorre na maioria dos sensores de proximidade (Siegwart et al., 2011).

Os sensores de proximidade são emulados neste trabalho de acordo com um alcance limitado de detecção, formando uma região circular em volta do robô, cujo o centro coincide com a posição atual da sua ARTag e possui um raio $D_{\text {Range }}$, que é fixo e determinado a partir de observações na capacidade de rastreamento das ARTags quando os robôs estão em movimento. Quando qualquer objeto detectável está no interior desta região de detecção, o robô é capaz de calcular a distância euclidiana entre sua posição atual e a do objeto. Quando um ou mais objetos são detectados desta forma, uma intensidade de iminência de colisão $([0,1])$ é atribuída a cada um, de acordo com a distância calculada anteriormente e normalizada pelo valor $D_{\text {Range }}$. Desta forma, uma intensidade próxima de 0 (zero) significa que o objeto detectado está distante do robô e não oferece risco de colisão. Por outro lado, quando a intensidade é próxima de 1 (um) indica que o objeto detectado está muito próximo da posição atual do robô e oferece um risco alto de colisão. Em resumo, os sensores emulados desta forma representam dispositivos periféricos acoplados à esquerda, à frente, atrás e à direita do robô.

Além disso, um robô utilizado nesta plataforma de experimentos é capaz de identificar se o objeto detectado é um alvo, um obstáculo estático e/ou outros robôs (obstáculos dinâmicos). Quando uma ARTag publicada faz parte da lista de alvos atribuida a um robô, ele identifica este marcador como um alvo e se aproxima deste, do contrário o robô considera o objeto (seja alvo ou outro robô) um obstáculo e implementa as manobras de desvio, de acordo com os comandos do controlador de locomoção. Ainda no caso do objeto identificado ser outro robô, é possivel a troca de informações entre eles.

Assim, a camada de realidade aumentada compreende a visualização de todos os marcadores virtuais em suas respectivas posições, juntamente com as habilidades que são conferidas virtualmente aos marcadores que representam os robôs. Esta camada virtual pode ser visualizada pelo computador por meio do pacote ROS denominado RViz ${ }^{6}$. Uma visão geral deste fluxo de informações é apresentado na Figura 3.

\section{DESCRIÇÃO DO SMR ANALISADO}

Nesta seção, a estratégia de planejamento de trajetória para múltiplos robôs apresentada em (Almeida et al.,

\footnotetext{
6 http://wiki.ros.org/rviz
} 


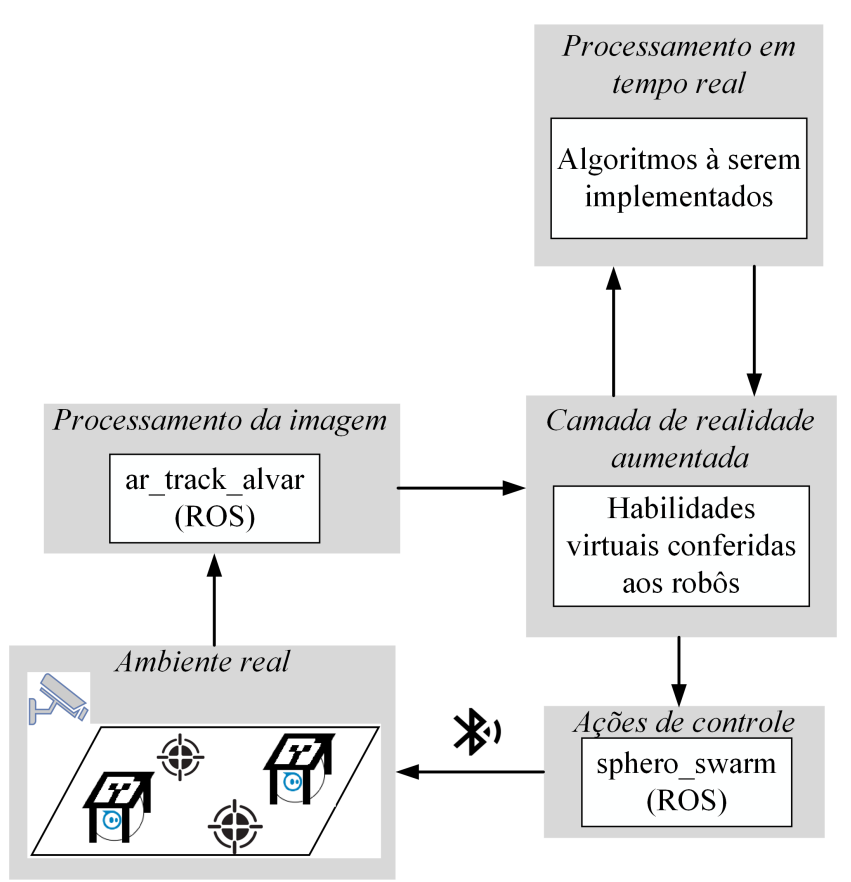

Figura 3. Fluxo de informação de um experimento na plataforma proposta.

2017), a qual foi unicamente validada por simulação, é adaptada para implementação na plataforma virtual.

De uma forma geral, o problema abordado neste exemplo pode ser ilustrado por meio da Figura 4, onde: 5 robôs estão em suas posições iniciais, há 20 alvos, 12 obstáculos estáticos e as linhas tracejadas são as trajetórias que os robôs devem desenvolver para atender os seguintes requisitos de navegação: $(i)$ visitar todos os alvos com as menores trajetórias possíveis; $(i i)$ evitar colisão entre os robôs (representam obstáculos dinâmicos no ambiente) e com os obstáculos estáticos; e (iii) finalizar a navegação em uma posição final.

$\mathrm{Na}$ plataforma proposta, a partir da filmagem inicial com identificação das posições inicial e final dos robôs e de todos os alvos a serem visitados, um conjunto de trajetórias ótimas é calculado pelo planejador de rotas desenvolvido em (Almeida et al., 2017) e atribuidas aos robôs na forma de lista de alvos (tópicos ROS) a serem visitados. No entanto, essas trajetórias não consideram a presença de obstáculos estáticos e/ou dinâmicos e, colisões podem ocorrer nas regiões destacadas pelos quadrados em cinza da Figura 4 (C1 a C5), durante a navegação.

O sistema de navegação utilizado para movimentar os robôs é o mesmo proposto em (Nakashima et al., 2018), o qual é composto por módulos fuzzy de controle. O principal objetivo deste sistema on-line é redirecionar o robô à sua trajetória planejada sempre que a rota divergir da calculada, devido às manobras de segurança necessárias para o desvio de obstáculos. As manobras de desvio e seguimento de trajetória sao implementadas pelo sistema de locomoção descrito na seção 2.2.

Para alcançar o objetivo mencionado, o robô verifica se o obstáculo mais próximo está à esquerda ou à direita de sua trajetória planejada e calcula um caminho secundário, por meio da rotação do vetor que interliga as posições

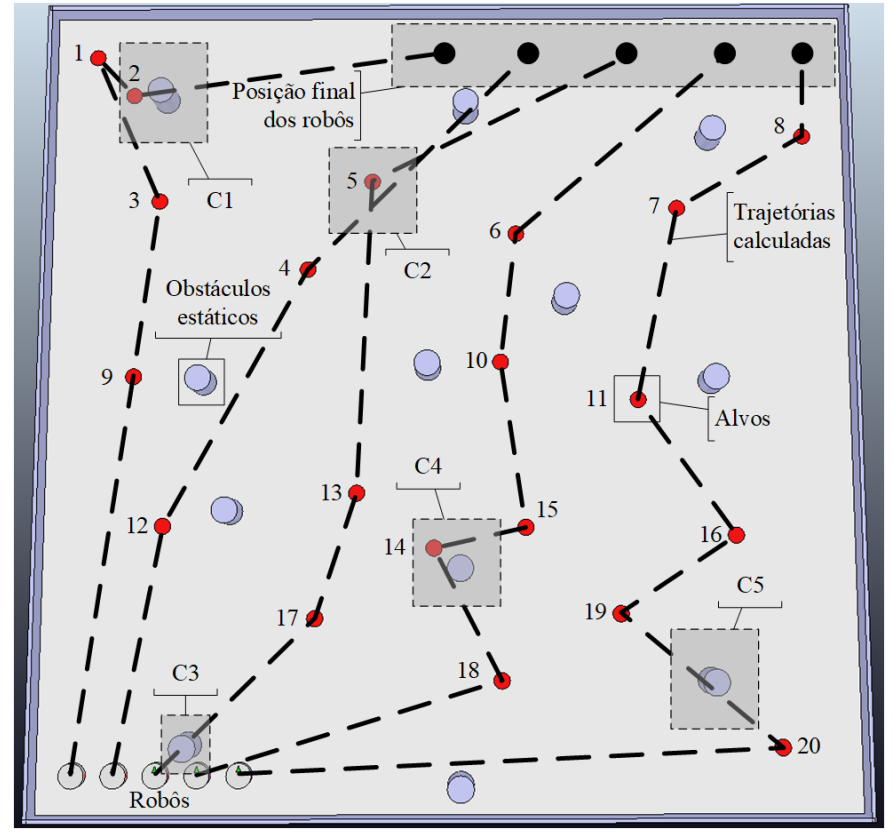

Figura 4. Ilustração do problema.

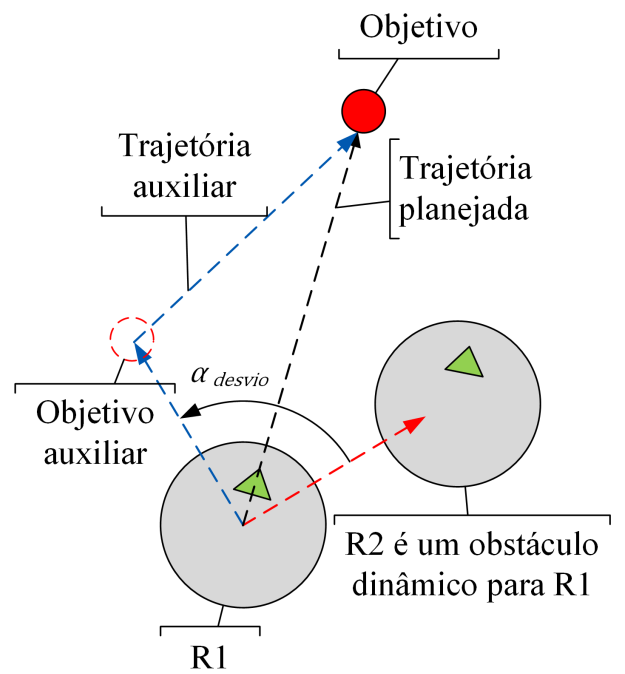

Figura 5. Manobra para correção de trajetória devido à presença de obstáculos.

atual do robô e seu próximo objetivo. Essa correção corresponde ao ângulo $\pm \alpha_{\text {desvio }}$, resultante das ações de controle combinadas e obtidas dos controladores fuzzy considerados. Este comportamento é exemplificado na Figura 5, onde o robô R1 detecta R2 como um obstáculo (indicado pela seta vermelha) e calcula a correção de sua trajetória, obtida por meio da inserção de um objetivo auxiliar, a partir do qual a trajetória planejada (seta preta) é substituída por uma auxiliar (seta azul).

\section{RESULTADOS EXPERIMENTAIS}

Nesta seção, o experimento real descrito em (Nakashima et al., 2018) será reproduzido, a fim de demonstrar o potencial da plataforma desenvolvida. Nesta análise, dois objetivos são traçados: $(i)$ verificar se os robôs são capazes de seguir suas trajetórias, calculadas anteriormente, 
enquanto desviam de obstáculos; e (ii) confirmar se a plataforma é capaz de atribuir as habilidades requeridas pelo sistema de navegação ao SMR, composto pelos robôs Sphero. Todo o experimento é acompanhado por meio da camada de realidade aumentada.

Nesta implementação é considerado um ambiente de tamanho $2,5 \times 1,5 m, 6$ alvos (A1 a A6) e 4 robôs (R1 a R4). As trajetórias são calculadas pelo método de planejamento de rotas desenvolvido em (Almeida et al., 2017). Na Figura 6 , três frames da navegação, obtidos de um vídeo gravado pela câmera USB, são mostrados na parte superior da figura e comparados com os frames RViz correspondentes (parte inferior da figura) que são disponíveis ao usuário pela plataforma desenvolvida. Na camada de realidade aumentada (frames RViz da parte inferior da Figura 6), cada elemento considerado no ambiente é diferenciado por cor (robôs em cores distintas, alvos em rosa e posições finais em azul) e o círculo ao redor de cada robô representa sua região de detecção de obstáculos. Para uma melhor compreensão, apenas as trajetórias planejadas (tracejado em azul) e executadas pelos robôs R1 (vermelho) e R4 (verde), incluindo as respectivas posições finais (quadrados em azul), são mostradas nesta figura.

De acordo com as trajetórias planejadas, R1 deve visitar apenas o alvo A4 e desviar de A3 (obstáculo para R1). Por outro lado, R4 deve visitar os alvos A5 e A6. Os frames 1 mostram a configuração inicial do experimento, quando todos os robôs estão alinhados na parte esquerda do ambiente. Esta condição no experimento real (frame 1) é corretamente reproduzida no ambiente virtual frame 1 (RViz). Após R4 visitar A5, há uma aproximação entre os robôs R1 e R3 no frame 2 real. No ambiente virtual, esta aproximação torna-se evidente pela superposição das áreas de detecção de ambos os robôs (frame 2 RViz) que determina o início de uma manobra de desvio entre eles. Após R1 e R4 visitarem os alvos A4 e A6, respectivamente, todos os robôs alcançam suas posições finais no lado direito do ambiente e o experimento é finalizado nos frames 3 , real e virtual.

Diante destes resultados, conclui-se que o objetivo $(i)$ da análise é confirmado, ou seja, os robôs seguem as trajetórias planejadas e realizam as manobras necessárias para o desvio de obstáculos. Em relação às funcionalidades da plataforma experimental (objetivo (ii)), observa-se que as habilidades de locomoção (controladores fuzzy) e de detecção (sensores de proximidade) são implementadas com êxito nos robôs, por meio de uma camada virtual, a qual também permite uma visualização "aumentada"da evolução do experimento.

\section{CONCLUSÕES}

Neste trabalho é proposta uma plataforma baseada em realidade aumentada, que suporta a navegação de múltiplos robôs em um ambiente real, em escala de laboratório. Para isso, uma câmera USB captura imagens do ambiente, a fim de detectar os marcadores ARTag que são associados aos objetos inseridos no cenário. Esta abordagem permite estabelecer um sistema de localização visual dos robôs e, ainda, emular dispositivos periféricos de sensoriamento (adicionais aos disponíveis ao usuário desta plataforma) e implementar virtualmente estratégias de controle não suportadas pelo hardware original dos robôs.

Uma descrição geral da plataforma é apresentada, ressaltando suas potencialidades, e um exemplo utilizando robôs do tipo Sphero, os quais apresentam severas limitações de hardware, é considerado para validar os recursos descritos.

Neste exemplo, múltiplos robôs Sphero, inseridos em um ambiente semi-desconhecido, tornam-se capazes de seguir trajetórias planejadas a priori que passam em pontos específicos (alvos) do ambiente e, durante a navegação, os robôs também desviam de obstáculos imprevistos e retomam as trajetórias planejadas após tais manobras.

As habilidades requeridas durante o experimento são conferidas virtualmente aos robôs pela plataforma, podendo ser avaliadas antes que um processo de prototipagem ou aquisição dos robôs e/ou dos dispositivos seja necessário.

A escalabilidade da plataforma proposta é limitada, principalmente, ao tamanho da área útil de experimentos, à capacidade de processamento da imagem obtida pela câmera (posição das ARTags) e pelo processamento centralizado da navegação dos robôs (computador com pacotes ROS).

Para trabalhos futuros, pretende-se aprimorar o sistema de detecção das ARTags, a fim de mitigar os problemas de perda de localização e de outras situações inesperadas (luminosidade na área experimental). Além disso, problemas de comunicação entre robôs (direta e indireta), deadlock, controle distribuído, entre outros, também serão investigados.

\section{REFERÊNCIAS}

Almeida, J.P.L.S., Arruda, L.V.R., and Neves-Jr, F. (2017). Planejamento de rota por meio de algoritmo genético para um enxame de robôs. In Simpósio Brasileiro de Automação Inteligente (SBAI 2017), 226-231.

Almeida, J.P.L.S., Nakashima, R.T., Neves-Jr, F., and Arruda, L.V.R. (2019). Bio-inspired on-line path planner for cooperative exploration of unknown environment by a multi-robot system. Robotics and Autonomous Systems, 112, 32 - 48. doi:https://doi.org/10.1016/j. robot.2018.11.005.

Fiala, M. (2005). Artag, a fiducial marker system using digital techniques. In 2005 IEEE Computer Society Conference on Computer Vision and Pattern Recognition (CVPR'05), volume 2, 590-596. doi:10.1109/ CVPR.2005.74.

Han, Z., Wang, D., Liu, F., and Zhao, Z. (2017). Multi-agv path planning with double-path constraints by using an improved genetic algorithm. PLOS ONE, 12(7), 1-16. doi:10.1371/journal.pone.0181747.

Hönig, W., Milanes, C., Scaria, L., Phan, T., Bolas, M., and Ayanian, N. (2015). Mixed reality for robotics. In 2015 IEEE/RSJ International Conference on Intelligent Robots and Systems (IROS), 1, 5382-5387. Hamburg. doi:10.1109/IROS.2015.7354138.

Millard, A.G., Redpath, R., Jewers, A.M., Arndt, C., Joyce, R., Hilder, J.A., McDaid, L.J., and Halliday, D.M. (2018). Ardebug: An augmented reality tool for analysing and debugging swarm robotic systems. Frontiers in Robotics and AI, 5, 87. doi:10.3389/frobt. 2018.00087 . 

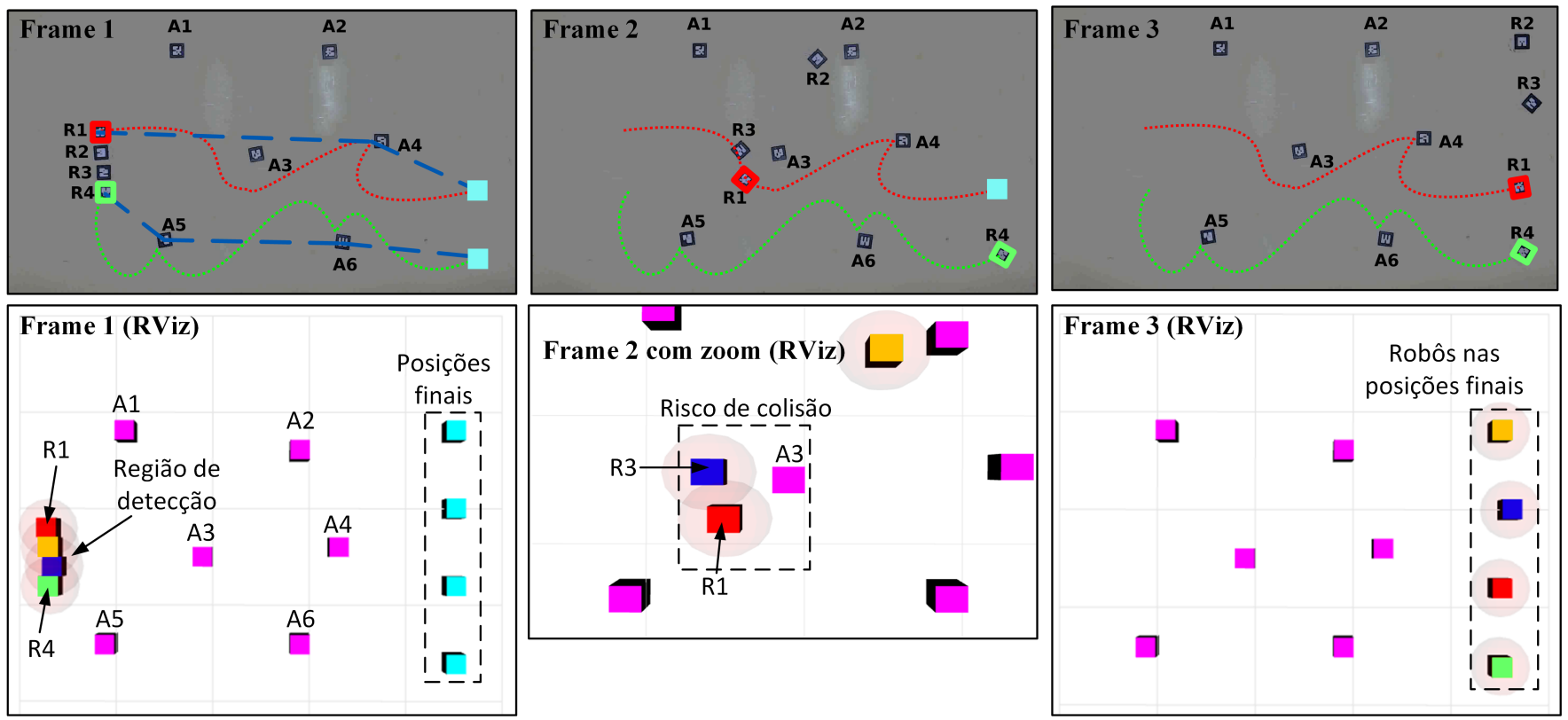

Figura 6. Frames de execução do exerimento real e sua vizualização em realidade aumentada.

Nakashima, R.T., Campera, B., Almeida, J.P.L.S., Arruda, L.V.R., and Neves-Jr, F. (2018). Uma arquitetura centralizada para navegação autônoma de múltiplos robôs sphero. In Congresso Brasileiro de Automática (CBA 2018), 1-8.

Reina, A., Cope, A.J., Nikolaidis, E., Marshall, J.A.R., and Sabo, C. (2017). Ark: Augmented reality for kilobots. IEEE Robotics and Automation Letters, 2(3), 17551761. doi:10.1109/LRA.2017.2700059.

Reina, A., Salvaro, M., Francesca, G., Garattoni, L., Pinciroli, C., Dorigo, M., and Birattari, M. (2015). Augmented reality for robots: Virtual sensing technology applied to a swarm of e-pucks. In $2015 N A S A / E S A$ Conference on Adaptive Hardware and Systems (AHS), 1-6. doi: 10.1109/AHS.2015.7231154.

Siegwart, R., Nourbakhsh, I.R., and Scaramuzza, D. (2011). Introduction to Autonomous Mobile Robots. The MIT Press, USA, 2nd edition.

Yanguas-Rojas, D. and Mojica-Nava, E. (2017). Exploration with heterogeneous robots networks for search and rescue. IFAC-PapersOnLine, 50(1), 7935 - 7940. doi: https://doi.org/10.1016/j.ifacol.2017.08.768. 